\title{
Comparative Study of Surface Interaction and Excitation
}

\author{
Abhishek Tiwari*1 \\ 1*. Department of Physics (Applied Sciences), SRIMT, DR APJ Abdul Kalam Technical University, Lucknow, (U.P.) 226201-India \\ e-mail : abhi.marut@gmail.com
}

\section{Publication Info}

\section{Article history :}

Received : $30^{\text {th }}$ March 2016

Accepted : $15^{\text {th }}$ June 2017

DOI : 10.18090/samriddhi.v9i01.8341

\section{Keywords :}

cnt's optical response, plasmons, polaritons.

*Corresponding author :

Abhishek Tiwari

e-mail : abhi.marut@gmail.com

\begin{abstract}
Extensive research has found nano-particles, for many years subject of unanimous concern because of their anomalous electromagnetic properties originating from the resonant interaction between light and collective conduction electron oscillations so called surface Plasmons. These properties in particular local electric field enhancements enable applications such as single molecule detection using surface enhanced Raman scattering and the synthesis of composite materials exhibiting an enhanced nonlinear optical response. Since all these applications rely on the resonant behavior of localized surface Plasmons, they are restricted to a limited frequency range determined by the dielectric function of the involved media, the size and shape of the particles and the electromagnetic interaction between them. Thus those properties of the Plasmons and Polaritons on the Carbon Nanotube have been studied in different size with help of theoretical study. The surface frequency of Plasmon varies with propagation constant on the surface of Carbon Nanotube when it is placed in dielectric medium, so this new property of Carbon Nanotube have been seen in other dielectric medium. This property of surface Plasmon can be used in telecommunication, satellite media, biomedical sciences etc.
\end{abstract}

\section{MODIFIED BESSEL FUNCTION}

A n infinitely long cylindrical wire of polar semiconductor of radius $\mathrm{R}$ in which $\mathrm{Z}$-axis coincides with the axis of the cylinder surrounded by a non-dispersive medium of dielectric constant $\varepsilon_{2}$ where the free electron concentration $n_{0}$ must satisfy the condition

$$
\begin{array}{cc}
n_{0}(\bar{r})=n_{0} & \mathrm{r}<\mathrm{R} \\
0 & \mathrm{r}>\mathrm{R}
\end{array}
$$

To solve the differential equation

$$
\left[\nabla^{2}-\alpha^{2}\right] n_{1}(\bar{r})=0
$$

Let the solution be

$$
n_{1}(r)=\sum_{l} n_{l}(\bar{r}) Y_{l}(\theta) e^{i(k, r-\omega t)} \text { for } \mathrm{k} \neq 0
$$

Eq. (1.2) can be written after putting the value of $n_{l}(r)$ from eq. (1.3)

Where $\quad \alpha^{2}=\frac{1}{\beta^{2}}\left[\omega_{p}^{2}-\omega^{2}\right]$

For cylindrical Harmonics

$$
\nabla^{2}=\frac{1}{r} \frac{\partial}{\partial r}\left(r \frac{\partial}{\partial r}\right)+\frac{1}{r^{2}}\left(\frac{\partial^{2}}{\partial \theta^{2}}\right)+\left(\frac{\partial^{2}}{\partial z^{2}}\right)
$$


The following graphs depicts that cnts are restricted to some limited frequencies of EMWs.
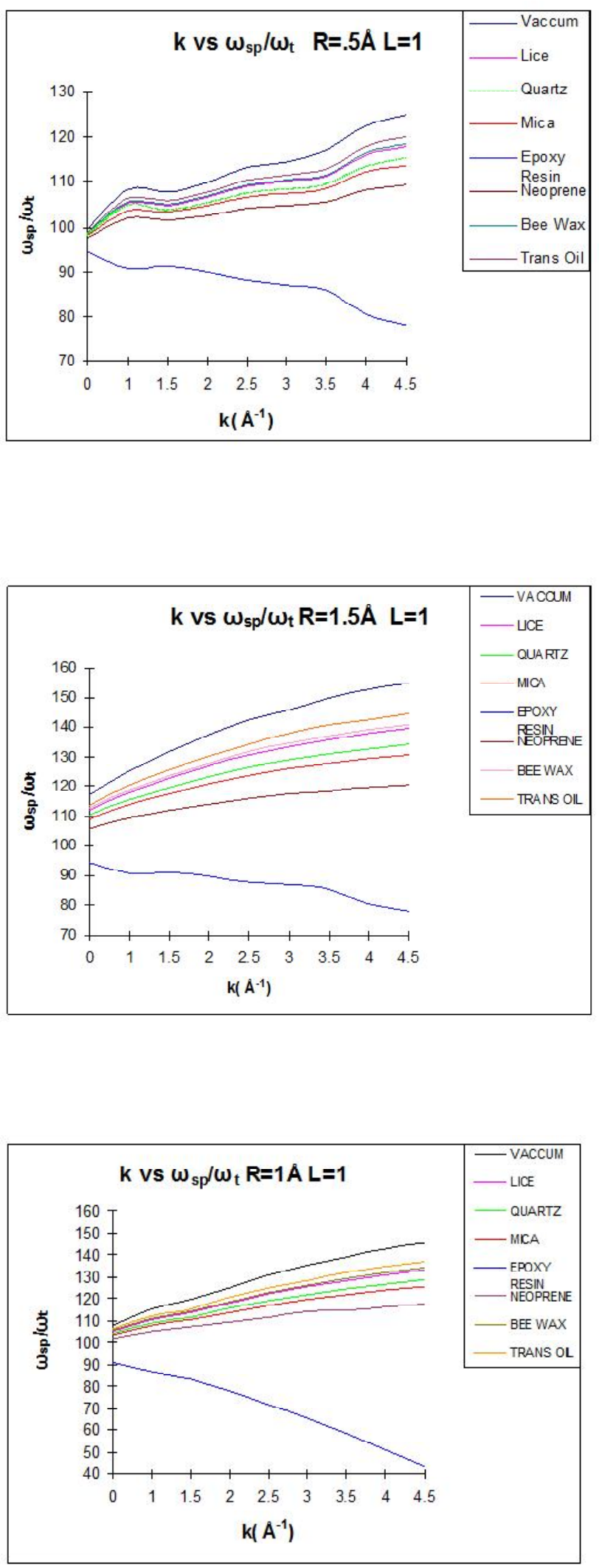

\section{CONCLUSION}

It shows clearly that carbon nanotube are placed in different medium the surface plasmon frequency varies with propagation constant $\mathrm{k}$. It predicts Epoxy resin is such a medium in which surface plasmon frequency decreases less than other medium with respect to propagation constant $\mathrm{k}\left(\AA^{-1}\right)$ because epoxy resin is insulated medium so there are no electron clouds in epoxy resin as EM wave incident on the CNT. Surface plasmon frequency increases but not so fast than other medium. It is also clear that for low value of $\mathrm{k} \omega_{\mathrm{sp}}$ is almost linear but as value of $\mathrm{k}$ increases $\omega_{\mathrm{sp}}$ almost decreases. At very high value of $\mathrm{k} \omega_{\mathrm{p}}$ almost decreases. On the other hand for medium lice and bee wax graph is exactly similar. This shows that both medium behaves as one single medium only for $\omega_{\mathrm{sp}}$ with respect to the propagation constant $\mathrm{k}$. there are very large variation in surface plasmon frequency with respect to the variation of propagation $\mathrm{k}$.

Therefore dispersion relation gives important information about surface of spherical polar semiconductor i.e. like filtering properties, a band attenuation high band gap\& low band gap.

The study serves a purpose for researchers and scientists to explore the material behavior exposed to electromagnetic radiation.

\section{REFERENCES}

[1] A. De. Martino, "An Introduction to the theory of Carbon nanotube", http://www.cs.infn.it/ de_martino_1.ppt, Accessed: 08/16/2005.

[2] Ritchie, R.H. 1957 Phy. Rev. 106, 874.

[3] Rivacoba, A. Apellp and Zabalan N 1995 Nuclear Instrument Meth B 96470 .

[4] Saito R., Eujita, G. Dresselhous G. and Dreseellhous M.S. 1992 Apll. Phys. Lett. 60,2204.

[5] Salvetat, J.P. Kulik, A.J. Briaas, G.A.D. Bonard J. M. Stockli, T. Burnham, N. and Forro L., 1999 Adv. Mater 11161. 